\title{
Efficacy of a Low-dose Subcutaneous Lisuride Infusion in Parkinson's Disease
}

\author{
Ryoichi Hayashi, Kenji Tako, Hideo Makishita, Jun Koyama and Nobuo Yanagisawa
}

\begin{abstract}
Five parkinsonian patients with motor fluctuations and dyskinesia after long-term treatment with levodopa were treated with subcutaneous lisuride infusion $(0.24-0.42 \mathrm{mg} / \mathrm{day})$ together with oral levodopa for a mean period of 27 (range 13-36) months. All 5 patients showed marked initial improvement in mobility. Mild psychiatric side effects were observed in three patients; however, these side effects disappeared with reduction in the dosage of lisuride to $0.06 \mathrm{mg}$ per day without a significant increase in motor fluctuations. A low dose of subcutaneous lisuride infusion with oral levodopa is an effective treatment for fluctuations of motor performance in parkinsonian patients without adverse psychiatric effects.

(Internal Medicine 37: 444-448, 1998)
\end{abstract}

Key words: motor fluctuation, dyskinesia, levodopa, dopamine agonist, clinical efficacy

\section{Introduction}

Daily fluctuations in motor performance, frequently accompanied by dyskinesia, are one of the most common problems in patients with Parkinson's disease after long-term treatment with levodopa (1). These serious adverse effects are particularly severe in patients with early onset of the disease (2). Continuous dopaminergic stimulation has been attempted to stabilize the motor fluctuations in severe parkinsonism. Levodopa and levodopa methyl-ester have been successfully used when administered intravenously $(3,4)$, by nasogastric route (5) or after gastrotomy (6). Treatment with dopamine agonists such as lisuride and apomorphine has also been attempted, intravenously or subcutaneously $(7,8)$. Clinical improvement resulting from subcutaneous lisuride infusion in some patients has been impressive; however, frequent neuropsychiatric reactions and dyskinesia have been the adverse side effects (8). In this paper, we report the use of low-dose subcutaneous lisuride infusion administered to five patients with severe parkinsonism after failing to obtain adequate control with standard therapy.

\section{Patients and Methods}

\section{Patients}

Five patients (four men and one woman) with Parkinson's disease were treated with subcutaneous lisuride given by continuous 24-hour infusion. All 5 patients showed severe wear- ing-off or on-off fluctuations unresponsive to control with conventional treatment (Table 1). Dyskinesia and dystonia were also deteriorative factors of disability in these patients. The criteria for patient selection were: 1) onset age under 45 years, 2) incapacitating fluctuations in motor performance or severe involuntary movements during oral levodopa treatment, and 3) absence of intellectual impairment or systemic diseases.

\section{Methods}

After informed consent was obtained, patients were admitted to hospital before starting lisuride infusion. Lisuride was given by continuous, subcutaneous infusion ( $24 \mathrm{~h}$ ). During lisuride infusion treatment, the dosage of levodopa was maintained without change.

Severity of parkinsonism was assessed by the Webster rating scale every two weeks. In addition, the patients were asked to record subjective disability score of their motor performance and involuntary movements on an hourly basis. The subjective score was based on the activities of daily living (ADL) and it was graded from " 0 " to " 5 ". Grades " 0 " and " 5 " were used to designate the best and the worst subjective disability which each patient had experienced before treatment with lisuride. Grade " 1 " was used to designate slight disability and grade " 3 " to indicate moderate. Grades " 2 " and " 4 " were used to indicate intermediate stages between grades " 1 " and " 3 " and between grades " 3 " and " 5 ". The mean subjective disability score was calculated as follows: each hourly score was averaged for a

From the Department of Medicine (Neurology), Shinshu University School of Medicine, Matsumoto

Received for publication July 10, 1997; Accepted for publication February 18, 1998

Reprint requests should be addressed to Dr. Ryoichi Hayashi, the Department of Medicine (Neurology), Shinshu University School of Medicine, Matsumoto, Nagano 390-0802 
Subcutaneous Lisuride Infusion in PD

Table 1. Clinical Characteristics of 5 Patients

\begin{tabular}{rccccccrr}
\hline Patient & Age & Sex & $\begin{array}{c}\text { Duration } \\
\text { of illness } \\
(\mathrm{yr})\end{array}$ & $\begin{array}{c}\text { Duration of } \\
\text { L-dopa therapy } \\
\text { (yr) }\end{array}$ & $\begin{array}{c}\text { Hoehn \& } \\
\text { Yahr } \\
\text { stage }\end{array}$ & $\begin{array}{c}\text { Type of } \\
\text { motor } \\
\text { fluctuation }\end{array}$ & Dyskinesia & L-dopa \\
\hline 1 & 46 & M & 14 & 5 & IV & on-off & biphasic & 400 \\
2 & 52 & M & 12 & 8 & IV & on-off & off period & 1,100 \\
3 & 44 & M & 8 & 5 & II & wearing off & biphasic & 650 \\
4 & 53 & F & 10 & 10 & IV & on-off & off period & 600 \\
5 & 51 & M & 13 & 10 & IV & on-off & off period & 1,000 \\
\hline
\end{tabular}

daily score, and then data for 10 to 30 days obtained from each patient for a month were used to calculate the monthly average disability score. The degree of dyskinesia was also graded from zero (no abnormality) to 5 (severe). Again grade " 1 " was used to designate slight dyskinesia and grade " 3 " to indicate moderate. Grades " 2 " and " 4 " were used to indicate intermediate stages between grades " 1 " and " 3 " and between grades " 3 " and "5". Routine biochemical tests of blood and urine, including hepatic and renal functions, were performed every 3 months. An electrocardiogram was recorded at initial assessment and at every 6 months.

\section{Subcutaneous Infusion}

Lisuride (Schering AG, Berlin, Germany) was diluted in saline for continuous ( $24 \mathrm{~h}$ ) subcutaneous administration using a portable mini-infusion pump. The pump was held inside an elastic belt worn around the abdomen. The initial amount of lisuride infused per day was $0.06 \mathrm{mg} / 24 \mathrm{~h}$ and was increased to $0.42 \mathrm{mg} / 24 \mathrm{~h}$, via an increase of $0.06 \mathrm{mg}$ every $2-5$ days. The lisuride solution was administered by means of a thin needle placed subcutaneously. This was replaced and its location was changed every 2 days. Patients were instructed to carry out all procedures by themselves.

\section{Results}

\section{Initial results}

Five patients were treated with continuous subcutaneous lisuride infusion, mean dose $0.34 \pm 0.08 \mathrm{mg} / 24 \mathrm{~h}$, added to oral levodopa ( $750 \pm 292 \mathrm{mg} /$ day). Improvements in mobility and dyskinesia were observed 3-4 days after starting subcutaneous lisuride infusion.

To investigate the correlation between levodopa and motor ability, the serum levels of levodopa were studied in three patients (patient numbers 1,2,3) before and 14 days after treatment with lisuride 0.3 to $0.42 \mathrm{mg}$ per day. In these three patients, the time needed to walk for $5 \mathrm{~m}$ and to turn over in bed shortened with small daily fluctuations, and the subjective disability scores improved continuously with the reduction of involuntary movements. However, there was no definite relationship established between these improvements and the concentration of serum levodopa (Fig. 1).

\section{Follow-up}

The five patients continued treatment with lisuride added to oral levodopa during a mean period of 27 months (range 13-36 months). Three of the 5 patients had psychiatric side effects after 5-11 months of lisuride treatment. Therefore, the amount of lisuride was reduced.

Figure 2 shows changes in both the rate on the Webster scale and the rate of dyskinesia during treatment with lisuride for the 5 patients. The rate on the Webster scale decreased after starting subcutaneous lisuride infusion and the optimal effects of lisuride were observed 2-3 months after initial treatment. Changes in ratings on the Webster scale correlated positively with the changes of the monthly average of the subjective disability score, but, not with changes in dyskinesia. Dyskinesia decreased for the first several months of lisuride treatment and then increased over the subsequent months of lisuride treatment.

For three patients (patient numbers 1,3,5) lisuride infusion was discontinued temporarily when the patient experienced psychiatric side effects. During suspension of lisuride treatment, their rating on the Webster scale worsened and fluctuated. After reinstatement of treatment with lisuride, even at a low dose $(0.06 \mathrm{mg} / \mathrm{day})$, the rating on the Webster scale for each patient improved or remained almost constant with the same dose of levodopa.

\section{Side effects}

The major complication was adverse psychiatric reactions. Three patients developed visual hallucinations and irritability during lisuride therapy at a relatively high dose. Following reduction in the dosage of lisuride, psychiatric reactions disappeared in these 3 patients. Throughout subcutaneous lisuride treatment, no patient complained of any gastrointestinal symptoms, orthostatic hypotension or edema which have been reported during treatment with oral lisuride (9). There were no abnormalities in routine biochemical tests of blood and urine, including hepatic and renal functions.

\section{Discussion}

Subcutaneous lisuride infusion reduced the levodopa-induced motor fluctuations and its efficacy was sustained over many months at a low dose, without severe side effects. Our 


\section{HAYASHI et al}

\section{Patient 1}

Patient 2

Patient 3

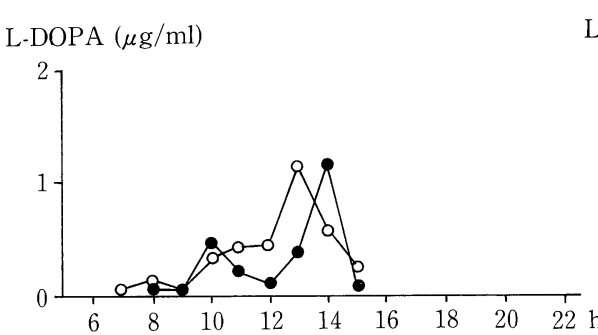

L-DOPA $(\mu \mathrm{g} / \mathrm{ml})$

L-DOPA $(\mu \mathrm{g} / \mathrm{ml})$
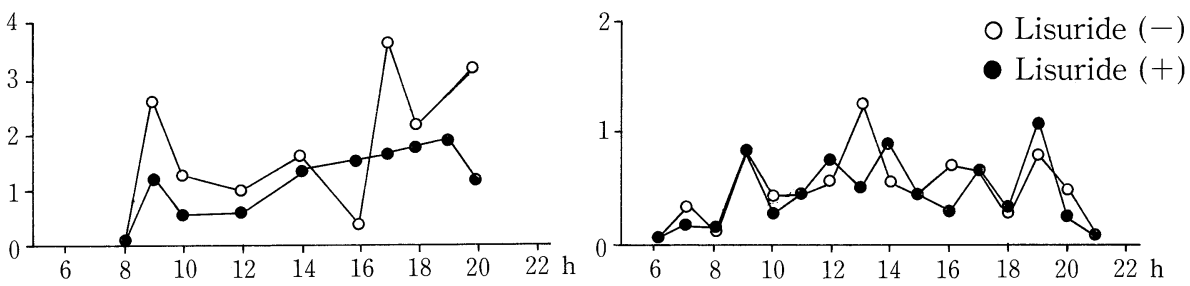

$5 \mathrm{~m}$ gait (sec)
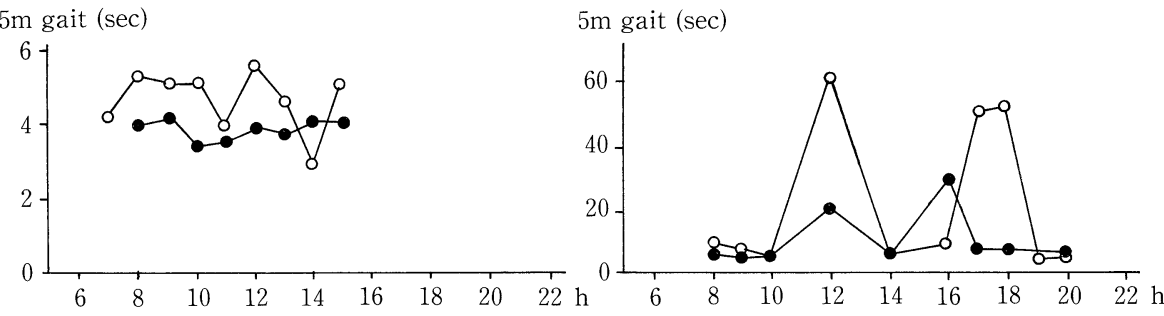

$5 \mathrm{~m}$ gait (sec)

Turn over $(\mathrm{sec})$

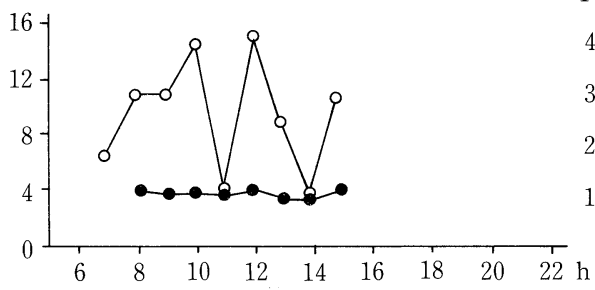

Turn over (sec)
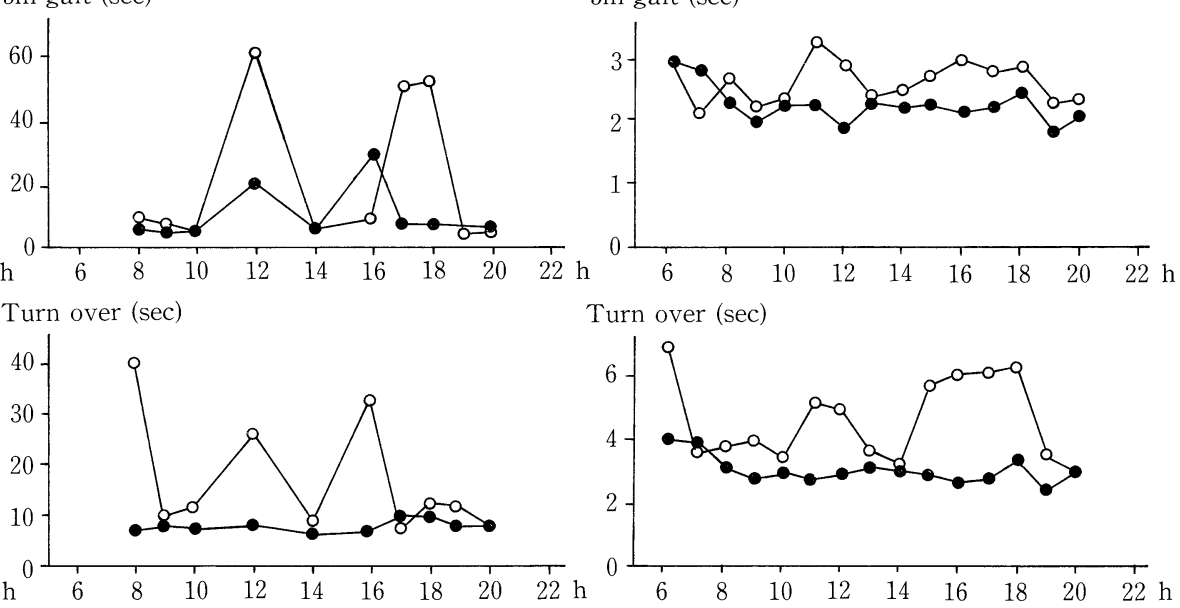

Subjective score

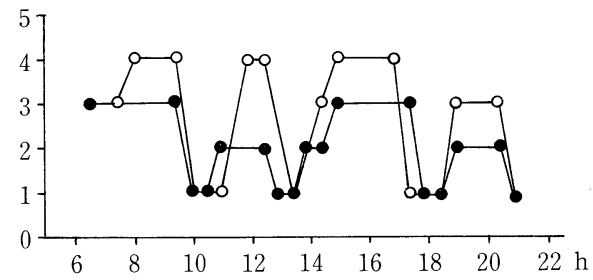

Subjective score
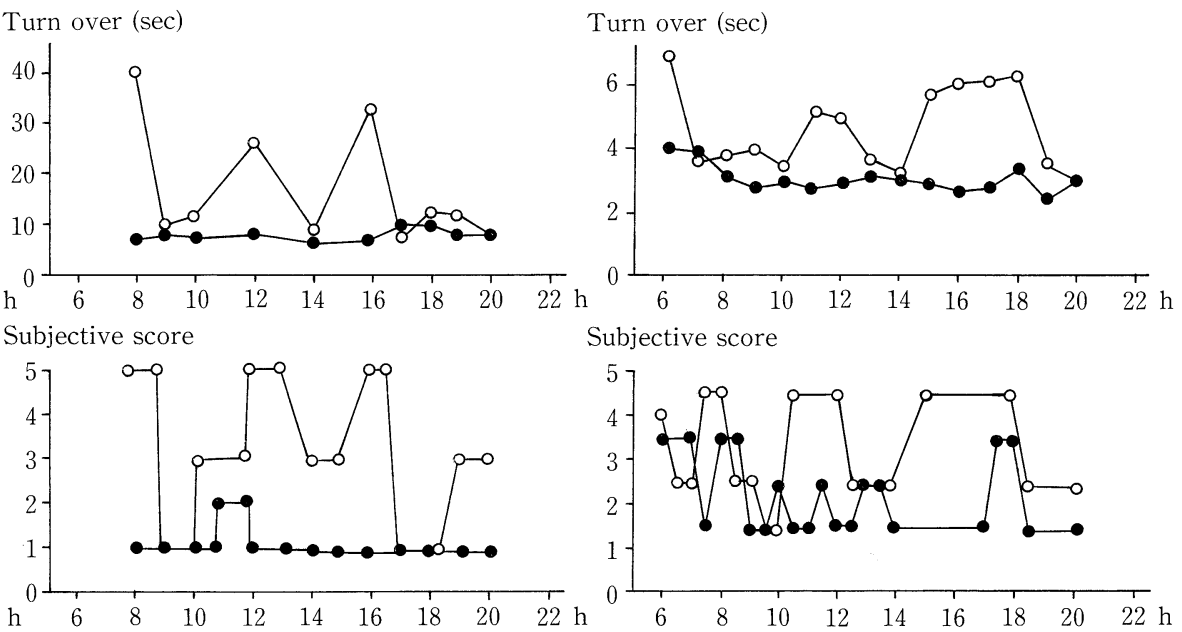

Subjective score

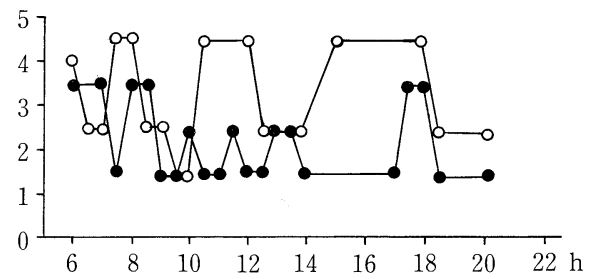

Figure 1. Efficacy of subcutaneous lisuride infusion on motor ability and subjective evaluation of daily activity. The relationship between the serum level of levodopa (L-dopa) and motor ability before and during lisuride treatment are shown. The motor ability was evaluated as the time required for the patient to walk $5 \mathrm{~m}$ and the time to turn over 360 degrees in bed. The subjective disability score was graded from zero (no abnormality) to 5 (severe). The open circles in each figure show data obtained before lisuride treatment. The time required for walking and turning over was shortened and the subjective scores improved when the serum levels of levodopa were high. The filled circles show the data obtained during treatment with lisuride $(0.3 \mathrm{mg} / 24 \mathrm{~h}$ for patient $1,0.42 \mathrm{mg} / 24 \mathrm{~h}$ for patients 2 and 3). The time required for walking and turning over shortened and the fluctuations of required times were reduced. The subjective disability scores also improved during lisuride treatment.

results also indicate that psychiatric reactions, occurring during lisuride treatment, were dependent on the amount of the dose of lisuride.

The adverse effects of levodopa treatment on patients with advanced Parkinson's disease are motor fluctuations due to a gradual shortening of the antiparkinsonian response to levodopa and to a progressive narrowing of its therapeutic window (10). The most significant clinical effect of subcutaneous lisuride infusion was the lessening of motor fluctuations $(8,11)$. In our study, continuous lisuride therapy ameliorated motor fluctuations without the necessity of changing the levodopa dose over a long term. Three patients experienced psychiatric side effects; therefore, lisuride infusion was temporarily suspended. During the suspension period, patients' ratings on the Webster scale worsened. After reinstatement of treatment with lisuride, even at a low dose, patient's ratings on the Webster scale remained almost constant. It has been suggested that lisuride acts on both presynaptic and postsynaptic dopaminergic receptor sites and that these effects lengthen the duration of effectiveness of levodopa and widen therapeutic windows (12). Our results showed that the improvement of motor ability was not related to the concentration of serum levodopa (cf., Fig. 1). Based on these findings, we conclude that lisuride infusion appears to provide a more beneficial physiological form of striate stimu- 
Patient 1

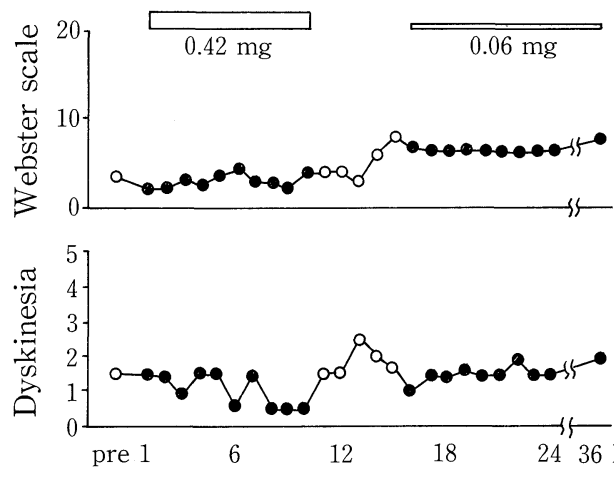

Patient 4
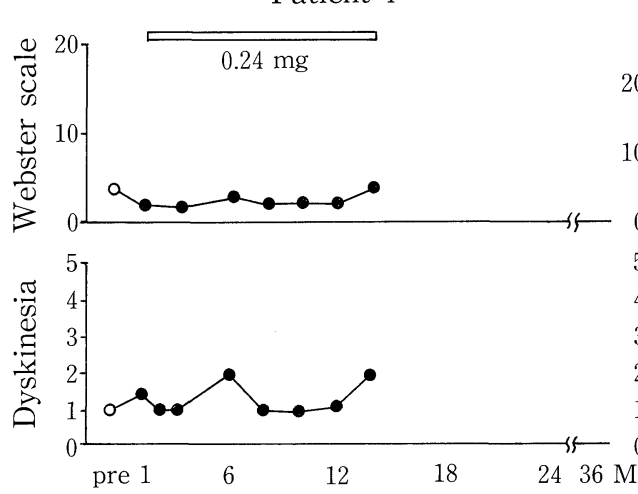

Figure 2. Long-term efficacy of subcutaneous lisuride infusion. Changes in rating on the Webster scale (upper panel) and the rate of dyskinesia (lower panel) for 5 patients are shown. Open circles indicate that patients were treated with only oral levodopa. Filled circles indicate that patients were treated with lisuride together with levodopa. Patients 2 and 4 were treated with a constant dose of lisuride, $0.30 \mathrm{mg}$ or $0.24 \mathrm{mg}$ per day, respectively. The other three patients were initially treated with lisuride, up to $0.42 \mathrm{mg}$ per day, and had subsequent side effects. Therefore, these patients were subsequently retreated with a low dose $(0.06 \mathrm{mg}$ per day).

lation to minimize motor incapacity during levodopa interdose periods.

Dyskinesia is a major troublesome complication in a significant proportion of cases treated with lisuride infusion. Dyskinesia during the 'off'/ 'on-off' period has been observed in about $70 \%$ of patients (8). In addition to the changes in motor response, adverse psychiatric reactions constituted another major drawback factor to the use of lisuride infusion. Several authors treated more than 10 PD patients for more than 3 months with $0.5-4.5 \mathrm{mg}$ per day, and observed a high incidence of psychiatric side effects in about $40 \%$ of patients $(8,11)$. Three of the present patients were freed from these side effects with the reduction of the dose of lisuride to $0.06 \mathrm{mg} / 24 \mathrm{~h}$. Therefore, the high incidence of psychiatric side effects may be directly related to the amount of lisuride.

The long-term efficacies and side effects of oral lisuride treatment were studied by Yanagisawa et al (9). They treated one hundred forty-five patients for 3 years with oral lisuride (1.1 $\pm 0.7 \mathrm{mg}$ per day) and oral levodopa. This regimen controlled $34 \%$ of patients well. However, they reported a high incidence of side effects: gastrointestinal symptoms (47\%) and psychiatric reactions $(18 \%)$. The present study was limited to only 5 patients; however, all 5 patients were controlled well for a long term period without these side effects with a small dose $(0.14 \pm$ $0.1 \mathrm{mg}$ per day), about $10 \%$ of the oral lisuride reported by Yanagisawa et al (9). Based on these findings, we conclude that continuous dopaminergic stimulation with a low dose of lisuride could provide optimal therapeutic results in reducing motor fluctuations without adverse side-effects.

Acknowledgements: The authors wish to thank Dr. R. Horowski for valuable comments.

\section{References}

1) Marsden CD, Parkes JD. "On-off" effects in patients with Parkinson's disease on chronic levodopa therapy. Lancet i: 292, 1976.

2) Narabayashi $H$, Yokochi M, Iizuka R, Nagatsu T. Juvenile parkinsonism. in: Handbook of Clinical Neurology Vol. 5 (49): Extrapyramidal disorders. Vinken PJ, Bruyn GW, Klawans HL, Eds. Elsevier Science Publishers B.V. Amsterdam, 1986, p. 153.

3) Marion MH, Stocchi F, Quinn NP, Jenner P, Marsden CD. Repeated levodopa infusions in fluctuating Parkinson's disease: clinical and pharmacokinetic data. Clin Neuropharmacol 9: 165, 1986.

4) Fabbrini G, Mouradian MM, Juncos JL, Schlegel J, Mohr E, Chase TN. Motor fluctuations in Parkinson's disease: central pathophysiological 


\section{HAYASHI et al}

mechanisms, part I. Ann Neurol 24: 366, 1988.

5) Kurlan R, Rubin AJ, Miller C, Rivera-Calimlim L, Clarke A, Shoulson I. Duodenal delivery of levodopa for on-off fluctuations in parkinsonism: preliminary observations. Ann Neurol 20: 262, 1986.

6) Sage JI, Trooskin S, Sonsalla PK, Heikkila R, Duvoisin RC. Long-term duodenal infusion of levodopa for motor fluctuations in parkinsonism. Ann Neurol 24: 87, 1988.

7) Stibe CMH, Lees AJ, Kempster PA, Stern GM. Subcutaneous apomorphine in parkinsonian on-off oscillations. Lancet i: 403, 1988.

8) Vaamonde J, Luquin MR, Obeso JA. Subcutaneous lisuride infusion in Parkinson's disease: response to chronic administration in 34 patients. Brain 114: 601, 1991.
9) Yanagisawa N, Kowa H, Mizuno Y, et al. Long-term study of lisuride maleate in patients with parkinsonism. Multi-center open study. J Med Pharmaceutical Sci 30: 1472, 1993.

10) Mouradian MM, Juncos JL, Fabbrini G, Schlegel J, Bartko JJ, Chase TN. Motor fluctuations in Parkinson's disease: central pathophysiologic mechanisms, part II. Ann Neurol 24: 372, 1988.

11) Obeso JA, Luquin MR, Vaamonde J, Martínez-Lage JM. Subcutaneous administration of lisuride in the treatment of complex motor fluctuations in Parkinson's disease. J Neural Transm Suppl 27: 17, 1988.

12) Baronti F, Mouradian MM, Davis TL, et al. Continuous lisuride effects on central dopaminergic mechanisms in Parkinson's disease. Ann Neurol 32: $776,1992$. 